frequency components seem to move in more or less the same direction lends validity to the wave hypothesis, and may be evidence that the waves are in fact produced by wind shear.

It must be stressed that the results given in Figs. 1 and 2, although typical of many occasions, are not universally observed. In many other cases the velocity of the Fourier components appeared to vary almost randomly with frequency, and it is assumed that in such cases the 'shallow sereen' approximation did not hold.

Scorer has examined the production of these surface gravity waves and has shown that they can be generated. by large-scale velocity shears in the atmosphere. He then uses these gravity waves to explain the production of billow clouds. In billow clouds, as in the present case, one wave-length predominates. The fact that sometimes one wave-length predominates implies that the spatial correlation function should be periodic with distance in the direction of drift. A large array of nine antennae has been used to show that this is in fact so.

It is concluded that 'drift' results may not always be interpreted as due to bodily motion of the ionospheric irregularities 'blown' by a wind. It appears probable that sometimes wave motion is the major factor in producing fading and it seems possible that these waves may be Helmholtz type surface gravity waves.

D. JONES

A. D. Maude

Department of Physics,

University College of Wales, Aberystwyth.

${ }^{1}$ Mitra, S. N., Proc. Inst. Elec. Eng., 96, 441 (1949).

2 Ratcliffe, J. A., and Pawsey, J. L., Proc. Camb. Phil. Soc., 29, 301 (1933).

'Helmholtz, H. von, Roy. Prussian Acad. of Sci., Berlin, Sitzungs-berichte, 761 (July 25, 1889).

4 Scorer, R. A., Archiv. fur Meteorol., Geophys. und Bioklimatol., 4, 176 (1953).

' Hines, C. O., Canad. J. Phys., 38, 1441 (1960).

- Hewish, A., Proc. Roy. Soc., A, 209, 81 (1951).

'Bowhill, S. A., Sci. Rep. No. 89, Ionosphere Res. Lab., University Park, Penn (1958).

- Goodman, N. R., Katz, S., Kramer, B. H., and Kuo, M. T., Technometrics, 3,245 (1961)

\section{Convergence and Divergence in Gravity and Magnetic Interpretation}

THe term average radial gravity $\bar{g}(r)$ may be defined as the arithmetic mean of all the gravity values lying on the circumference of a circle of radius $r$. A similar definition holds good in the magnetic case also. This can be represented as a series expansion in terms of the even vertical derivatives at the centre as:

$$
\bar{g}(r)=a_{0}+a_{2} r^{2}+a_{4} r^{4}+a_{6} r^{6}+\ldots
$$

where $a_{2}, a_{4}, a_{6}, \ldots$ are closely connected with the even vertical derivatives at the centre by the relations:

$$
\frac{\partial^{2} g}{\partial z^{2}}=-4 a_{2}, \frac{\partial^{4} g}{\partial z^{4}}=64 a_{4} \text { and } \frac{\partial^{6} g}{\partial z^{6}}=-2,304 a_{6}
$$

and $a_{0}$ approximates the gravity value at the centre.

Assuming that the series converges rapidly (Peters 1949, Elkins 1951, etc.), the terms related to higher derivatives and therefore higher powers of $r$ are omitted in the expansion for practical purposes. But it can be easily shown that the series is not always absolutely convergent and its convergence or divergence depends on the radius of the circle chosen. The series is convergent only up to a limiting value of $r=R$, in which case the sum of all the terms in the series tends to $\bar{g}(r)$. For values of $r>R$, the series becomes oscillatory and the sum of the terms in the series oscillates between $+\infty$ and $-\infty$. On the other hand, if the series is equated to an expression of finite number of terms by omitting terms containing higher powers of $r$, the sum of the terms diverges from $\bar{g}(r)$, for values of $r>R$, and the direction of divergence depends on the number of terms included.

A rigorous mathematical proof for the foregoing statement is, however, complicated and therefore only a qualitative technique has been adopted. As an example we can take the simple case of a sphere, for which the average radial gravity vertically above its centre can be given by:

$$
\bar{g}(r)=\frac{K}{z^{2}}-\frac{6 K r^{2}}{4 z^{4}}+\frac{120 K r^{4}}{64 z^{6}}-\frac{5040 K r^{6}}{2304 z^{8}}
$$

When only the first four terms are taken into account.

The foregoing expression will therefore converge if Lt $A_{n}=0$ and each term is less than its preceding,

or $A_{n} / A_{n-1}<1$; otherwise it diverges from $\bar{g}(r)$. Three ratios can be obtained in this case:

$$
\left|\frac{A_{2}}{A_{1}}\right|=\frac{3}{2}\left(\frac{r}{z}\right)^{2},\left|\frac{A_{3}}{A_{2}}\right|=\frac{5}{4}\left(\frac{r}{z}\right)^{2} \text { and }\left|\frac{A_{4}}{A_{3}}\right|=\frac{7}{6}\left(\frac{r}{z}\right)^{2}
$$

Each of the foregoing ratios and therefore the average of the three should be less than unity in order that the expression should converge to $\bar{g}(r)$. Taking the average of the ratios, the series is therefore convergent if $(r / z)^{2}<36 / 47$, or $R=0.88 Z$. For all other values of $R>0.88 \mathrm{Z}$, the series diverges. It can be clearly seen that the radius $R=0.88 Z$, at which the expression begins to diverge, is linearly related to the depth.

Similar results are obtained in the cases of gravity and magnetic anomalies of other geometrical forms also. Similar theory applies for bodies of irregular shape and for points not lying above the centre. In such a case the theory is complicated. However, it can be safely concluded that the expansion is only conditionally convergent and diverges from a particular value of $r=R$, which is related to the depth, the relation being dependent on the nature of the field, the number of the terms chosen and other factors.
B. S. R. RAO
I. V. RADHAKRISHNAMURTY

Geophysics Department, Andhra University, Waltair, India.

\section{GEOLOGY}

\section{Possible South-westward Continuations of the Cabot Fault}

Recentuy, Wilson ${ }^{1}$ directed attention to the Cabot (Palaeozoic) fault through Newfoundland, the Bay of Fundy, and along the New England coast. He compared it tentatively with the San Andreas of California and the Great Glen of Scotland. My colleagues and $\mathrm{I}^{2-5}$ have suggested similar faults of sub-parallel trend to the south-westward.

This communication directs attention to the fact that some of these hypothecated features lie along possible, and even probable, south-eastward trends of the Cabot fault. I lay no claim to having detailed either the literature or the field between these suggested features. I have, however, investigated available published maps and think that a summary of my preliminary thinking may be of assistance to other workers in guiding future investigations.

In the Gulf of Maine, there are three faults shown by Wilson ${ }^{1}$, the trends of which are not clearly enough indicated on his small-scale sketch map to permit choice with any degree of certainty at present (Fig. 1). I think, nevertheless, that it is desirable, at this time, to point out the possibilities that have occurred to me. In so 